\title{
Edge-State Velocity and Coherence in a Quantum Hall Fabry-Pérot Interferometer
}

\section{Citation}

McClure, Douglas T., Yiming Zhang, Eli M. Levenson-Falk, Charles M. Marcus, Loren N. Pfeiffer, and Kenneth W. West. 2009. Edge-state velocity and coherence in a quantum hall Fabry-Pérot interferometer. Physical Review Letters 103(206806).

\section{Published Version}

doi:10.1103/PhysRevLett.103.206806

\section{Permanent link}

http://nrs.harvard.edu/urn-3:HUL.InstRepos:5110748

\section{Terms of Use}

This article was downloaded from Harvard University's DASH repository, and is made available under the terms and conditions applicable to Open Access Policy Articles, as set forth at http:// nrs.harvard.edu/urn-3:HUL.InstRepos:dash.current.terms-of-use\#OAP

\section{Share Your Story}

The Harvard community has made this article openly available.

Please share how this access benefits you. Submit a story.

Accessibility 


\title{
Edge-State Velocity and Coherence in a Quantum Hall Fabry-Pérot Interferometer
}

\author{
D. T. McClure, ${ }^{1}$ Yiming Zhang, ${ }^{1}$ B. Rosenow, ${ }^{1,2}$ E. M. \\ Levenson-Falk, ${ }^{1}$ C. M. Marcus, ${ }^{1}$ L. N. Pfeiffer, ${ }^{3}$ and K. W. West ${ }^{3}$ \\ ${ }^{1}$ Department of Physics, Harvard University, Cambridge, Massachusetts 02138, USA \\ ${ }^{2}$ Max-Planck-Institute for Solid State Research, Heisenbergstr. 1, D-70569 Stuttgart, Germany \\ ${ }^{3}$ Bell Laboratories, Alcatel-Lucent Technologies, Murray Hill, New Jersey 07974, USA
}

(Dated: March 29, 2009)

\begin{abstract}
We investigate nonlinear transport in electronic Fabry-Pérot interferometers in the integer quantum Hall regime. For interferometers sufficiently large that Coulomb blockade effects are absent, a checkerboard-like pattern of conductance oscillations as a function of dc bias and perpendicular magnetic field is observed. Edge-state velocities extracted from the checkerboard data are compared to model calculations and found to be consistent with a crossover from skipping orbits at low fields to $\vec{E} \times \vec{B}$ drift at high fields. Suppression of visibility as a function of bias and magnetic field is accounted for by including energy- and field-dependent dephasing of edge electrons.
\end{abstract}

The electronic Fabry-Pérot interferometer (FPI), implemented as a quantum dot in the quantum Hall $(\mathrm{QH})$ regime, has attracted theoretical [1, 2, 3, 4, [5] and experimental [6, 7, 8, 9, 10, 11] interest recently, especially in light of the possibility of observing fractional [1] or non-Abelian 2, 3, 5, 12, statistics in this geometry. Earlier experiments reveal that Coulomb [13, 14, 15] and Kondo [16, 17] physics can play important roles, as well. With such a rich spectrum of physics in these devices, a thorough understanding of the mechanisms governing transport even in the integer $\mathrm{QH}$ regime remains elusive.

While most work on electronic FPI's to date has focused on transport at zero dc bias, finite-bias measurements have proved to be a useful tool in understanding the physical mechanisms important in other interferometer geometries. In metallic [18] and semiconducting [19] rings interrupted by tunnel barriers, oscillations in transmission as a function of magnetic field and dc bias, forming a checkerboard pattern, have been observed. These features, attributed to the electrostatic Aharonov-Bohm (AB) effect [20, 21, 22, were used to measure the time of flight and dephasing in these devices. Similar checkerboard-like lobe structures have also been observed in Mach-Zehnder interferometers [23, 24, 25]. In that case, the pattern of oscillations is not readily explained within a single-particle picture and remains the subject of continued theoretical study [26, 27, 28, 29]. In electronic FPI's, conductance oscillations as a function of dc bias have been investigated theoretically [1 and provide a means of extracting the edge-state velocity from the period in dc bias. Edge-state velocity measurement without the use of high-bandwidth measurements 30, 31] will likely be useful in determining appropriate device parameters to probe exotic statistics beyond the integer regime. This approach was recently used [7] to measure the edge-state velocity at $\nu=1 / 3$, though in a small $\left(\sim 1 \mu \mathrm{m}^{2}\right)$ device where Coulomb interactions, absent in the theory, may be expected to play a dominant role [9, 11].

In this Letter, we present measurements of finite-bias conductance oscillations in an $18 \mu \mathrm{m}^{2}$ electronic FPI whose zero-bias behavior is consistent with $\mathrm{AB}$ interference without significant Coulomb effects [11. We find a checkerboard-like pattern of conductance oscillations as a function of dc bias and magnetic field, in agreement with the predictions of Chamon et al. 1 1. Measuring the period in dc bias allows the velocity of the tunneling edge state to be extracted over a range of magnetic fields, yielding a low-field saturation consistent with a crossover from $\vec{E} \times \vec{B}$ drift to skipping orbits. Highbias fading in the checkerboard pattern is quantitatively consistent with a dephasing rate proportional to energy and magnetic field. Zero-bias oscillations in a $2 \mu \mathrm{m}^{2}$ device of similar design, where Coulomb effects are significant [1], do not evolve periodically with dc bias; instead, plots of conductance versus bias and magnetic field reveal diamond-like regions of blockaded transport in the weakforward-tunneling regime that become more smeared out with stronger forward tunneling.

Devices are fabricated on GaAs/AlGaAs quantum-well structures with a two-dimensional electron gas (2DEG) of density $n=2.7 \times 10^{15} \mathrm{~m}^{-2}$ and mobility $\mu=$ $2,000 \mathrm{~m}^{2} / \mathrm{Vs}$ located $200 \mathrm{~nm}$ below the surface. Hall bars are wet-etched as shown in Fig. 1(a), and metal surface gates are patterned by electron-beam lithography as in Fig. 1(b). Interferometers are defined by negative voltages $(\sim-3 \mathrm{~V})$ applied to all gates except $V_{\mathrm{C}}$, and samples are cooled in a dilution refrigerator to $\sim 20 \mathrm{mK}$. A current bias $I$, consisting of a dc component of up to $30 \mathrm{nA}$ and a $135-\mathrm{Hz}$ component of $400 \mathrm{pA}$, gives rise to the diagonal voltage $V_{\mathrm{D}}$ across the device, measured directly across the width of the Hall bar [Fig. 1(a)]. Lock-in measurements of diagonal conductance, $G_{\mathrm{D}} \equiv d I / d V_{\mathrm{D}}$, are used to study changes in interferometer transmission as a function of both $V_{\mathrm{D}}$ and perpendicular magnetic field $B$. As shown in Fig. 1(c), the current-carrying chiral edge states can be partially reflected at each constriction, leading to interference between the different possible trajectories as a function of the phase accumulated 


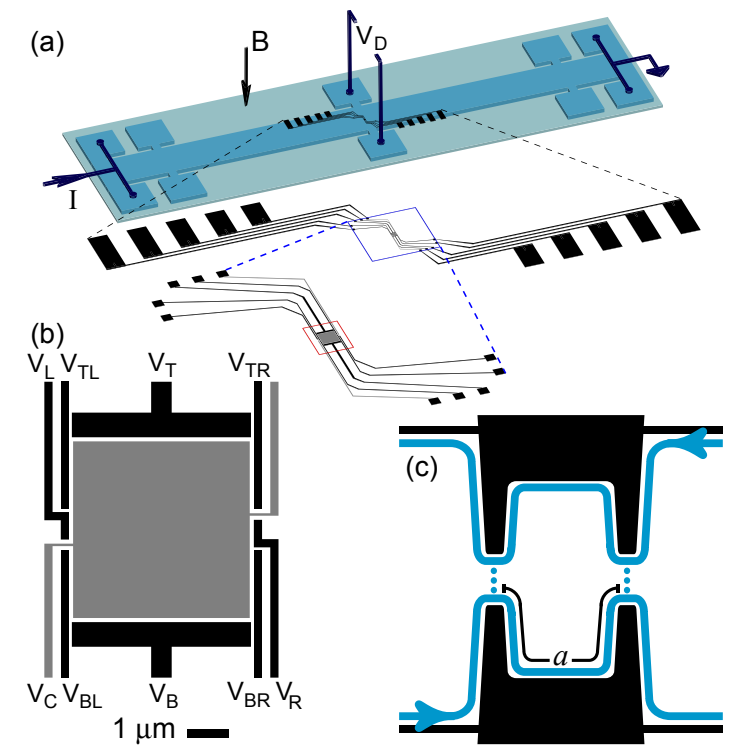

FIG. 1: (a) With a current bias $I$ applied at one end of the Hall bar, voltage $V_{\mathrm{D}}$ is measured directly across its width. Surface gates are shown in increasing detail, with a red box indicating the region shown in (b). (b) Gate layout of the $18 \mu \mathrm{m}^{2}$ device, which is operated as an interferometer by depleting all gates except $V_{\mathrm{C}}$. (c) Schematic diagram of possible transmission paths through the device in the quantum Hall regime.

by encircling the interferometer.

A typical measurement of $G_{\mathrm{D}}$ as a function of $B$ and $V_{\mathrm{D}}$ in the $18 \mu \mathrm{m}^{2}$ device is shown in Fig. 2(a), where a smooth background has been subtracted. A checkerboard-like pattern of oscillations periodic in both $B$ and $V_{\mathrm{D}}$ is observed, with reduced amplitude at high bias. Similar patterns are seen at fields $B=0.22-1.26 \mathrm{~T}$; over this range the Landau level index, $N$, of the tunneling edge ranges from 4 to 1 , but the field period of oscillations is always $\Delta B \approx 0.25 \mathrm{mT}$, independent of both field and bias.

Magnetoconductance oscillations in this device reflect $\mathrm{AB}$ interference of partially transmitted edge states [1], with a phase shift $\Delta \varphi=2 \pi \Phi / \Phi_{0}$, where $\Phi=B A$ is the flux enclosed (in area $A$ ) by the interfering edge, and $\Phi_{0} \equiv h / e$ is the magnetic flux quantum. The observed field period corresponds to $A \approx 17 \mu \mathrm{m}^{2}$, consistent with the dot area after subtracting a depletion length of roughly the 2DEG depth. The sinusoidal lineshape of the oscillations seen here suggests that coherent transport is dominated by two trajectories that differ in length by one traversal of the dot perimeter.

When a dc bias is added to $V_{\mathrm{D}}$, an additional phase shift appears between interfering trajectories, associated with the energy-dependent wave vector of the contributing edge-state electrons; we will refer to this as the FabryPerot phase. The wave vector changes with energy as $\delta k=\delta \varepsilon / \hbar v$, where $v$ denotes the edge-state velocity. Following the analysis of non-interacting electrons in Ref. [1], in which bias is assumed to affect mainly the chemical
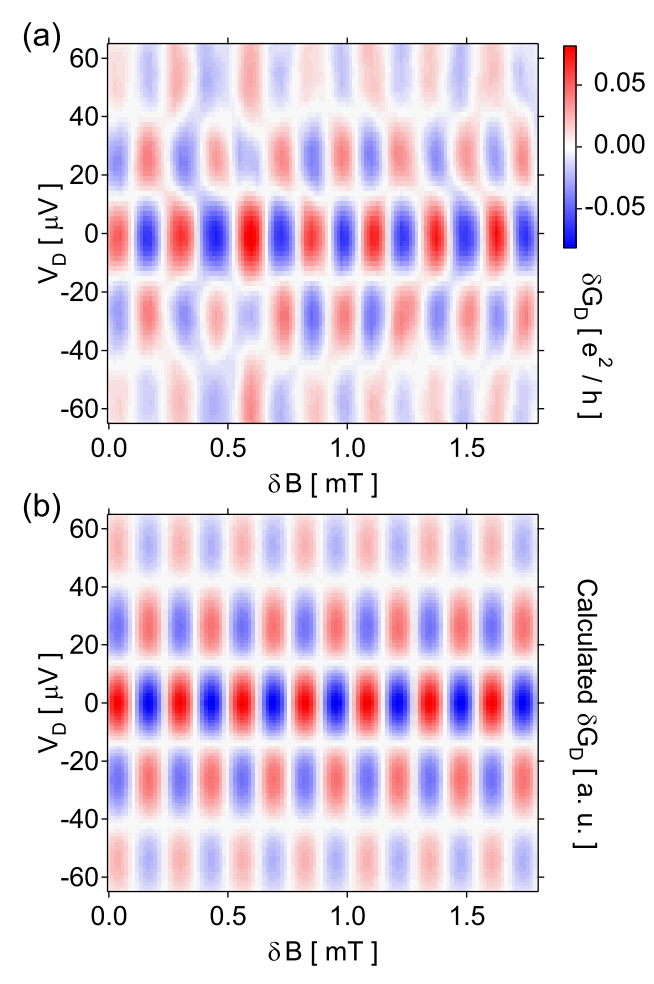

FIG. 2: (a) $G_{\mathrm{D}}$ as a function of $B$ and $V_{\mathrm{D}}$ in the $18 \mu \mathrm{m}^{2}$ device near $B=0.47 T$, with a smooth background subtracted. (b) $\delta G_{\mathrm{D}}$ calculated from Eq. (1), multiplied by the damping factor from Eq. (2), with $\Delta B=0.25 \mathrm{mT}, \Delta V_{\mathrm{D}}=56 \mu \mathrm{V}$, and $\alpha=0.2$.

potential, we assign an additional relative phase $2 a \varepsilon / \hbar v$ to an electron traversing the perimeter at energy $\varepsilon$ above the zero bias Fermi level, where $a \sim 2 \sqrt{A}=8.2 \mu \mathrm{m}$ denotes the path length between constrictions [Fig. 1(c)]. For a symmetrically applied dc bias (relative to the gate voltages), and neglecting contributions from multiplyreflected trajectories, the expected differential conductance has the form

$$
\delta G_{\mathrm{D}}\left(\Phi, V_{\mathrm{D}}\right)=\delta G_{0} \cos \left(2 \pi \Phi / \Phi_{0}\right) \cos \left(e V_{D} a / v \hbar\right),
$$

where the amplitude $\delta G_{0}$ does not depend on field or $\mathrm{dc}$ bias. Note that in this model, the contributions of $\mathrm{AB}$ and Fabry-Pérot phase separate into a product of two cosines, yielding a checkerboard pattern, as observed in the experimental data, Fig. 2(a). Ref. [1] predicts that when the bias is only applied to one contact, with the other contact held at ground (again, relative to the gates), the two phase contributions from bias and field instead appear as arguments of a single cosine, yielding a diagonal stripe pattern. Experimentally, the bias is always applied only at one end of the Hall bar, with the other end grounded; however, interaction effects within the dot are likely to effectively symmetrize the applied bias [32. Alternatively, a model in which the bias mainly affects the electrostatic (rather than chemical) potential [33] also yields Eq. (1) without the need for a sym- 
metric bias. In either interpretation, the bias period corresponds to the edge velocity via $\Delta V_{\mathrm{D}}=(h / e)(v / a)$.

We account for the reduced amplitude of oscillations at high bias by multiplying the right side of Eq. (1) by a damping factor, $e^{-2 \pi \alpha\left|V_{\mathrm{D}}\right| / \Delta V_{\mathrm{D}}}$, where $(2 \pi \alpha)^{-1}$ gives the number of periods over which the amplitude falls to $1 / e$ of its zero-bias value. Lacking theory for edge-state dephasing in FPI's, this form is motivated by the observation in related experiments of a dephasing rate proportional to energy [19, 34]. We thus identify a voltagedependent dephasing rate, $\tau_{\varphi}^{-1}\left(V_{\mathrm{D}}\right)=\alpha\left|e V_{\mathrm{D}}\right| / 2 \hbar$, which reduces amplitude by $e^{-2 t_{\circ} / \tau_{\varphi}}$, where $2 t_{\mathrm{o}}=2 a / v$ is the time of flight around the interferometer. To extract interference and dephasing parameters, the form

$$
\delta G\left(V_{\mathrm{D}}\right)=\delta G_{0} e^{-2 \pi \alpha|\delta x|} \cos (2 \pi \delta x),
$$

where $\delta x=\left(V_{\mathrm{D}}-V_{\text {off }}\right) / \Delta V_{\mathrm{D}}$ and $V_{\text {off }}$ is a bias offset, is fit to cuts of the data in Fig. 2(a), which yields a period $\Delta V_{\mathrm{D}}=56 \mu \mathrm{V}$ and dephasing parameter $\alpha=0.2$. These values, along with $\Delta B=0.25 \mathrm{mT}$ are then used to produce the plot shown in Fig. 2(b). Figures 3(a) and 3(b) show vertical cuts from data along with fits of Eq. (2) at $B=0.22 \mathrm{~T}$ and $1.26 \mathrm{~T}$, respectively, representing a trend toward smaller $\Delta V_{\mathrm{D}}$ and larger $\alpha$ at higher fields, the details of which we now study.

The black circles in Fig. 3(c) indicate the best-fit $\Delta V_{\mathrm{D}}$ (right axis) and corresponding edge velocity (left axis) as a function of $1 / B$. The velocities appear roughly proportional to $1 / B$ before saturating at $v \sim 1.5 \times 10^{5} \mathrm{~m} / \mathrm{s}$ for $1 / B \gtrsim 2 T^{-1}$. Red curves indicate calculations based on single-particle models of edge velocities in two regimes. In the high-field limit, where the cyclotron radius is much smaller than the length scale on which the confining potential changes by the cyclotron gap, $\vec{E} \times \vec{B}$ drift gives a velocity $v_{\mathrm{d}}=E / B$, where $E$ is the local slope of the confining potential. The data in this regime are consistent with a value $E \sim 8 \times 10^{4} \mathrm{~V} / \mathrm{m}$, which is reasonable given device parameters. At low fields, where the cyclotron radius exceeds the length scale set by $E$, electron velocities can be estimated from a skipping-orbit model. For hardwall confinement, the skipping velocity would be proportional to the cyclotron frequency and radius: $v_{s} \sim \omega_{c} r_{c}$. Here, we have performed a detailed semi-classical calculation assuming a more realistic confining potential that vanishes in the bulk and grows linearly near the edge. In this regime, the predicted velocity depends on not only $B$ and $E$ but also on the Landau level index, $N$, resulting in a discrete jump in velocity for every change in $N$. Since the density in the constrictions (which along with $B$ determines $N$ ) varies over the course of the experiment, two theoretical curves are plotted in this regime: the top one corresponds to the lowest observed constriction density of $2.8 \times 10^{14} \mathrm{~m}^{-2}$, and the bottom one corresponds to the highest, $9.5 \times 10^{14} \mathrm{~m}^{-2}$, both estimated from $G_{\mathrm{D}}$ and $B$.

Figure 3(d) shows the best-fit damping parameter $\alpha$ as a function of $B$, revealing rough proportionality: a
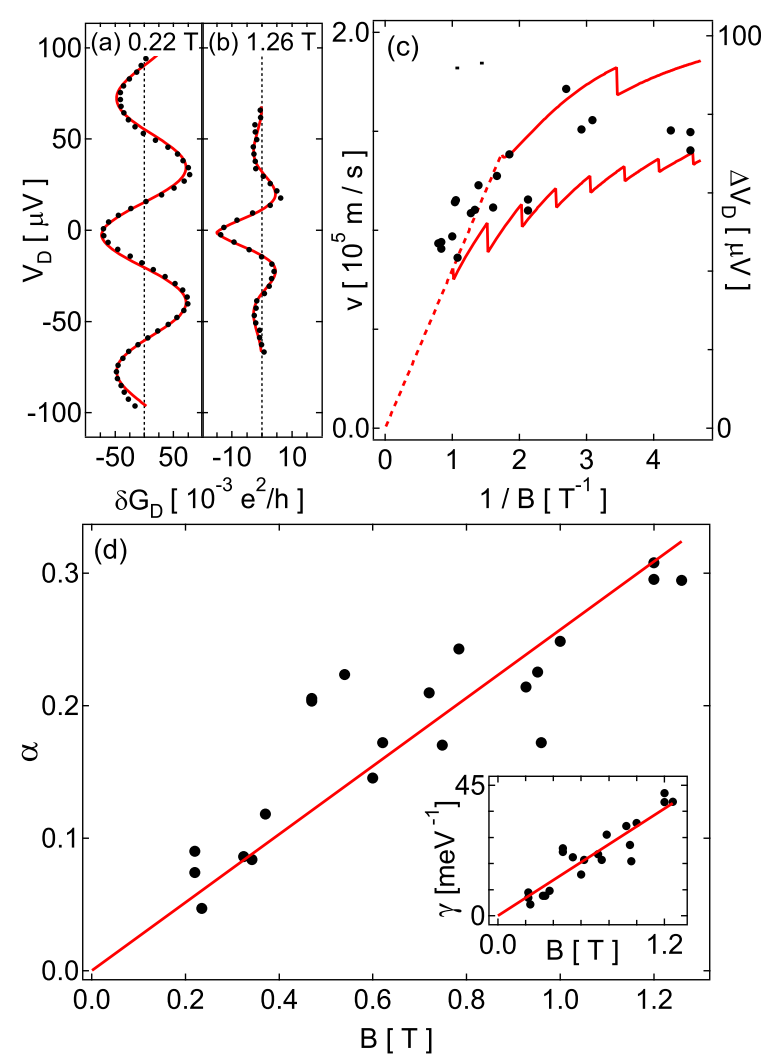

FIG. 3: (a) $G_{\mathrm{D}}$ as a function of $V_{\mathrm{D}}$ (black dots) at a field of $B=$ $0.22 \mathrm{~T}$, with a fit of Eq. (2) (red curve) yielding $\Delta V_{\mathrm{D}}=76 \mu \mathrm{V}$ and $\alpha=0.063$. (b) Same as (a) but at $B=1.26 \mathrm{~T}$ and yielding $\Delta V_{\mathrm{D}}=$ $47 \mu \mathrm{V}$ and $\alpha=0.34$. (c) Black dots indicate edge velocities (left axis) determined from measured $\Delta V_{\mathrm{D}}$ (right axis) as a function of $1 / B$. The red curves indicate theoretical calculations: at low $1 / B$, the diagonal dashed line indicates the drift velocity corresponding to $E=8 \times 10^{4} \mathrm{~V} / \mathrm{m}$; at high $1 / B$, the top and bottom solid curves indicate the predicted skipping-orbit velocities corresponding to the lowest and highest constriction densities, respectively. (d) Best-fit damping parameter $\alpha$ as a function of $B$, with a linear fit of slope $0.26 \mathrm{~T}^{-1}$ constrained through the origin. Inset: $\gamma=2 \pi \alpha / e \Delta V_{\mathrm{D}}$ as a function of $B$, with a linear fit of slope $31(\mathrm{meV} \cdot \mathrm{T})^{-1}$ constrained through the origin.

straight line constrained to cross the origin describes the data well with a best-fit slope of $0.26 \mathrm{~T}^{-1}$. In analogy to dephasing in 2D diffusive systems [35, we suggest that coupling to compressible regions in the bulk may lead to dephasing with the $V_{D}$-dependence $\tau_{\varphi}^{-1} \propto R_{\square} V_{D}$, where $R_{\square}$ is the resistance per square in the bulk. Over the field range of our data, the bulk longitudinal resistivity $R_{x x}$ (not shown) is on average roughly proportional to $B$; taking $R_{x x}$ as an estimate of $R_{\square}$ would then lead to a predicted dephasing rate proportional to both energy and magnetic field, consistent with the data. Despite this agreement, we emphasize that Ref. [35] was not developed for edge states or FPI's, and a theory of dephasing in this regime remains lacking.

Alternatively, defining the damping factor as simply $e^{-\gamma\left|e V_{D}\right|}$, one also finds rough proportionality between $\gamma$ 
and $B$, as shown in the inset of Fig. 3(d). Here the bestfit slope for a straight line constrained through the origin is $31(\mathrm{meV} \cdot \mathrm{T})^{-1}$. The damping parameter $\gamma$ is related to $\alpha$ and to the dephasing length, $\ell_{\varphi}=v \tau_{\varphi}$, by $\gamma=\alpha t_{\mathrm{o}} / \hbar=$ $2 a /\left|e V_{D}\right| \ell_{\varphi}$; therefore, since $t_{\mathrm{o}}$ varies with field, at most one of $\alpha$ and $\gamma$ can be proportional to B. Physically, the latter case would correspond to $\ell_{\varphi}^{-1}$ being the quantity that is linear in $B$ instead of $\tau_{\varphi}^{-1}$. Experimental scatter prevents us from distinguishing these two possibilities.

Measurements on a $2 \mu \mathrm{m}^{2}$ device of similar design, whose zero-bias oscillations have previously been demonstrated as consistent with Coulomb-dominated behavior [1], do not yield regular oscillations as a function of bias. Figure 4 shows $G_{\mathrm{D}}$ as a function of $B$ and $V_{\mathrm{D}}$ in a regime of weak forward-tunneling, where diamondlike features appear. Interpreting these features as the result of Coulomb blockade yields a charging energy of roughly $25 \mu \mathrm{eV}$, reasonable given the device size, 2DEG depth, and the large capacitance afforded by the top gate. In regimes of stronger forward tunneling, the diamond edges become more smeared out, but in contrast to the behavior in the $18 \mu \mathrm{m}^{2}$ device, periodic oscillations as a function of dc bias are not seen.

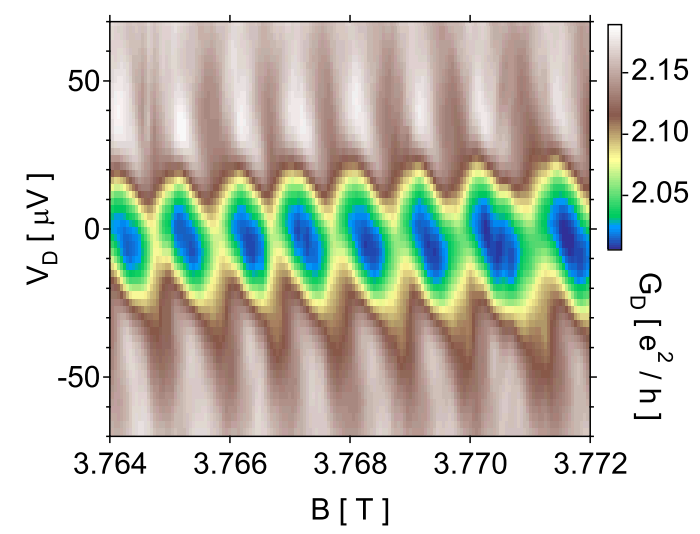

FIG. 4: $G_{\mathrm{D}}$ as a function of $B$ and $V_{\mathrm{D}}$ in the $2 \mu \mathrm{m}^{2}$ device.

In conclusion, quantum Hall FPI's large enough that Coulomb charging is negligible are found to display both $\mathrm{AB}$ and Fabry-Pérot conductance oscillations. The combination of these two effects yields a checkerboard-like pattern of oscillations from which the edge-state velocity and dephasing rate can be extracted, and both are found to be consistent with theoretical calculations. Although this pattern resembles that seen in Mach-Zehnder interferometers, the dependence of its characteristics on magnetic field is evidently quite different from what has been observed in those devices [23, 25], providing experimental evidence that the underlying mechanisms for oscillations with bias in the two types of devices may be quite different.

We are grateful to R. Heeres for technical assistance and to B. I. Halperin, M. A. Kastner, C. de C. Cha- mon, A. Stern, I. Neder, R. Gerhardts, J. B. Miller and I. P. Radu for enlightening discussions. This research has been funded in part by Microsoft Corporation Project Q, IBM, NSF (DMR-0501796), Harvard University, and the Heisenberg program of DFG. Device fabrication at Harvard Center for Nanoscale Systems.

[1] C. de C. Chamon et al., Phys. Rev. B 55, 2331 (1997).

[2] P. Bonderson, A. Kitaev, and K. Shtengel, Phys. Rev. Lett. 96, 016803 (2006).

[3] A. Stern and B. I. Halperin, Phys. Rev. Lett. 96, 016802 (2006).

[4] B. Rosenow and B. I. Halperin, Phys. Rev. Lett. 98, 106801 (2007).

[5] R. Ilan, E. Grosfeld, and A. Stern, Phys. Rev. Lett. 100, 086803 (2008).

[6] F. E. Camino, W. Zhou, and V. J. Goldman, Phys. Rev. Lett. 95, 246802 (2005).

[7] F. E. Camino, W. Zhou, and V. J. Goldman, Phys. Rev. B 74, 115301 (2006).

[8] F. E. Camino, W. Zhou, and V. J. Goldman, Phys. Rev. Lett. 98, 076805 (2007).

[9] M. D. Godfrey et al. (2007), arXiv:0708.2448.

[10] R. L. Willett et al. (2008), arXiv:0807.0221.

[11] Y. Zhang et al. (2009), arXiv:0901.0127.

[12] C. Nayak et al., Rev. Mod. Phys. 80, 1083 (2008).

[13] B. J. van Wees et al., Phys. Rev. Lett. 62, 2523 (1989).

[14] B. W. Alphenaar et al., Phys. Rev. B 46, 7236 (1992).

[15] P. L. McEuen et al., Phys. Rev. B 45, 11419 (1992).

[16] M. Keller et al., Phys. Rev. B 64, 033302 (2001).

[17] M. Stopa et al., Phys. Rev. Lett. 91, 046601 (2003).

[18] A. van Oudenaarden et al., Nature 391, 768 (1998).

[19] W. G. van der Wiel et al., Phys. Rev. B 67, 033307 (2003).

[20] Y. Aharonov and D. Bohm, Phys. Rev. 115, 485 (1959).

[21] Y. V. Nazarov, Phys. Rev. B 47, 2768 (1993).

[22] Y. V. Nazarov, Physica B 189, 57 (1993).

[23] I. Neder et al., Phys. Rev. Lett. 96, 016804 (2006).

[24] P. Roulleau et al., Phys. Rev. B 76, 161309 (2007).

[25] L. V. Litvin et al., Phys. Rev. B 78, 075303 (2008).

[26] E. V. Sukhorukov and V. V. Cheianov, Phys. Rev. Lett. 99, 156801 (2007).

[27] J. T. Chalker, Y. Gefen, and M. Y. Veillette, Phys. Rev. B 76, 085320 (2007).

[28] I. P. Levkivskyi and E. V. Sukhorukov, Phys. Rev. B 78, 045322 (2008).

[29] I. Neder and E. Ginossar, Phys. Rev. Lett. 100, 196806 (2008).

[30] R. C. Ashoori et al., Phys. Rev. B 45, 3894 (1992).

[31] G. Ernst et al., Physica E 1, 95 (1998).

[32] B. I. Halperin, private communication.

[33] B. Rosenow, B. I. Halperin, and A. Stern, unpublished.

[34] P. Roulleau et al., Phys. Rev. Lett. 100, 126802 (2008).

[35] B. L. Altshuler and A. G. Aronov, in Electron-Electron Interactions in Disordered Systems, edited by A. L. Efros and M. Pollak (North-Holland, Amsterdam, 1985). 